\title{
Selectivity of Interaction of Phospholipids with Bovine Spinal Cord Myelin Basic Protein Studied by Spin-Label Electron Spin Resonance
}

\author{
M. B. Sankaram, ${ }^{\ddagger}$ Peter J. Brophy, ${ }^{\S}$ and Derek Marsh, ${ }^{* \ddagger}$ \\ Max-Planck-Institut für biophysikalische Chemie, Abteilung Spektroskopie, D-3400 Göttingen, Federal Republic of Germany, \\ and Department of Biological Science, University of Stirling, Stirling FK9 4LA, United Kingdom \\ Received February 13, 1989; Revised Manuscript Received July 18, 1989
}

\begin{abstract}
The selectivity of interaction between bovine spinal cord myelin basic protein (MBP) and eight different spin-labeled lipid species in complexes with dimyristoylphosphatidylglycerol (DMPG) and between spin-labeled phosphatidylglycerol and spin-labeled phosphatidylcholine in complexes of MBP with various mixtures of DMPG and dimyristoylphosphatidylcholine (DMPC) has been studied by electron spin resonance (ESR) spectroscopy. In DMPC/DMPG mixtures, the protein binding gradually decreased with increasing mole fraction of DMPC in a nonlinear fashion. The lipid-protein binding assays indicated a preferential binding of the protein to phosphatidylglycerol relative to phosphatidylcholine without complete phase separation of the two lipids. The outer hyperfine splittings $\left(2 A_{\max }\right)$ of both phosphatidylglycerol and phosphatidylcholine labeled at C-5 of the $s n-2$ chain (5-PGSL and 5-PCSL, respectively) were monitored in the lipid-protein complexes as a function of the mole fraction of DMPC. The increases in the value of $A_{\max }$ induced on binding of the protein were larger for 5-PGSL than for 5-PCSL, up to 0.25 mole fraction of DMPC. Beyond this mole fraction the spectral perturbations induced by the protein were similar for both lipid labels. The ESR spectra of phosphatidylglycerol and phosphatidylcholine labeled at C-12 of the $s n-2$ chain were two component in nature, indicating a direct interaction of the protein with the lipid chains, at mole fractions of DMPC up to 0.25 . Quantitation of the motionally restricted spin-label population by spectral subtraction again indicated a preferential interaction of the protein with phosphatidylglycerol relative to phosphatidylcholine. Up to DMPC mole fractions of 0.25 , the microenvironment of the protein was enriched in DMPG. The interaction of MBP with phosphatidylcholine (PC), phosphatidylethanolamine (PE), phosphatidylglycerol (PG), phosphatidylserine (PS), phosphatidic acid (PA), phosphatidylinositol (PI), diphosphatidylglycerol (CL), and diacylglycerol (DG), spin-labeled on C-5 of the sn-2 chain, was studied in complexes with DMPG. The protein-induced increases in the outer hyperfine splitting of the spin-label ESR spectra established a sequence for the selectivity of interaction with the MBP in the order $\mathrm{PS}^{-}>\mathrm{CL}^{-}$ $>\mathrm{PA}^{2-}>\mathrm{PG}^{-}>\mathrm{PI}^{-}>\mathrm{PA}^{-}>\mathrm{PE}^{ \pm}>\mathrm{PC}^{ \pm}>\mathrm{DG}$. Assuming fast exchange for the ESR spectra of the C-5 labels, relative association constants of the different lipids with the MBP were determined. $\mathrm{pH}$ titration of the PA spin-label in DMPG complexes revealed a stronger interaction with the protein of the doubly negatively charged than of the singly charged species, and the shift in the $\mathrm{p} K$ of the PA spin-label indicated a partial dehydration of the DMPG lipid surface on binding of the protein.
\end{abstract}

$T_{\text {he }}$ nerve axon is enveloped by the oligodendroglial cell plasma membrane which allows for an efficient sequestering of the intra- and extracellular contents [see Braun (1977) and Rumsby and Crang (1977)]. Lipid-protein interactions are important determinants of the insulating properties of the nerve membrane that stabilize the multilayered structure of the myelin sheath. Among the myelin proteins, the myelin basic protein $(\mathrm{MBP})^{1}$ attracts special attention due to its large molecular area which presumably leads to a sealing of the membrane surface, thereby hindering transmembrane molecular exchange. The molecular composition of the myelin membrane is heterogeneous in both lipid and protein [see Boggs and Moscarello (1978a) and Boggs et al. (1982)]. The peripheral basic protein and an integral proteolipid protein constitute nearly $80 \%$ (by weight) of the total proteins. In addition to cholesterol, different but significant amounts of cerebrosides, phospholipids (phosphatidylcholines, phosphatidylethanolamines phosphatidylserines, and phosphatidylinositols), and sphingomyelin constitute the lipid fraction of this membrane (Rumsby, 1978).

\footnotetext{
${ }^{\ddagger}$ Max-Planck-Institut.
}

University of Stirling
Several physicochemical studies [Surewicz et al., 1987; Boggs et al., 1985, 1986; Ramsay et al., 1986; also see Boggs and Moscarello (1978a)] on the protein conformation and lipid-protein interactions have led to important insights into the molecular mechanism of the function of MBP. The MBP has no detectable tertiary structure in aqueous solutions (Eylar \& Thompson, 1969; Chao \& Einstein, 1970; Palmer \& Dawson, 1969) but adopts $\alpha$-helical and $\beta$-sheet conformations in nonpolar environments like organic solvent mixtures (Liebes et al., 1975; Stone et al., 1985), micelles (Mendz et al., 1984), and lipid bilayers (Surewicz et al., 1987). A strong binding to acidic lipids (Palmer \& Dawson, 1969) has been observed. Although binding to lipid bilayers is predominantly electro-

\footnotetext{
${ }^{1}$ Abbreviations: ESR, electron spin resonance; DMPG, 1,2-dimyristoyl-sn-glycero-3-phosphoglycerol; DMPC, 1,2-dimyristoyl-snglycero-3-phosphocholine; EDTA, ethylenediaminetetraacetic acid; Tris, tris(hydroxymethyl)aminomethane; $n$-PGSL, -PASL, -PSSL, -PISL, PESL, -PCSL, 1 -acyl-2-[n-(4,4-dimethyl- $N$-oxyoxazolidine) stearoyl]sn-glycero-3-phosphoglycerol, -phosphoric acid, -phosphoserine, -phosphoinositol, -phosphoethanolamine, -phosphocholine; 5-CLSL, 1-(3-snphosphatidyl)-3-[1-acyl-2-[4-(4,4-dimethyl-3-oxy-2-tridecyl-2-oxazolidinyl)butanoyl]-sn-glycero(3)phospho]-sn-glycerol; 5-DGSL, 1-acyl-2[4-(4,4-dimethyl-3-oxy-2-tridecyl-2-oxazolidinyl)butanoyl]-sn-glycerol; MBP, bovine spinal cord myelin basic protein.
} 
static in nature, hydrophobic interactions that presumably arise from a partial penetration of the protein into membranes have been detected by using differential scanning calorimetry (Papahadjopoulos et al., 1975), nuclear magnetic resonance (Mendz et al., 1984), and spin-label electron spin resonance (Boggs \& Moscarello, 1978b; Sankaram et al., 1989) spectroscopic techniques.

Recently, it has been shown that the MBP binds strongly to dimyristoylphosphatidylglycerol (DMPG) bilayers and exhibits saturation binding (Sankaram et al., 1989). Spin-label ESR spectroscopic studies on DMPG-MBP complexes using phosphatidylglycerol spin-label positional isomers have shown that approximately $50 \%$ of the bound lipids are motionally restricted by the protein. It is of considerable biological relevance to extend these studies to mixtures with zwitterionic lipids, mimicking the heterogeneous lipid composition of the native myelin membrane, and also to investigate the relative specificities of interaction with the different phospholipids composing the native membrane. We report here our results on the binding of the MBP to mixed bilayers of dimyristoylphosphatidylcholine and dimyristoylphosphatidylglycerol with systematically varying composition and on the relative selectivities of interaction of phosphatidylcholine and phosphatidylglycerol in these mixed bilayers. The selectivities for interaction with the myelin basic protein of a wide range of spin-labeled phospholipids, encompassing analogues of the phospholipids composing the myelin membrane, have also been investigated in bilayers of pure DMPG. Combined with studies of the $\mathrm{pH}$ titration behavior, these results give a more detailed picture of the phospholipid properties determining the MBPlipid interaction and its modulation by the lipid composition of the membrane. In particular, a high specificity is found for phosphatidylserine, one of the two major negatively charged lipids of the myelin membrane.

\section{Materials AND Methods}

Materials. The myelin basic protein was isolated from bovine spinal cord as described by Deibler et al. (1972). DMPG was prepared from DMPC (Fluka, Buchs, Switzerland) by a headgroup exchange reaction (Comfurius \& Zwaal, 1977 ) catalyzed by phospholipase D (Boehringer-Mannheim, FRG). 5-DGSL was synthesized from 5-PCSL by a phospholipase $C$ (Boehringer-Mannheim) catalyzed hydrolysis reaction (Zwaal et al., 1971), 5-PISL was synthesized from yeast phosphatidylinositol (Lipid Products, South Nutfield, U.K.) by using a modification for spin-labeled lipids of the method described by Somerharju and Wirtz (1982). 5-CLSL was synthesized by a condensation reaction between 5-PGSL and phosphatidic acid derived from egg yolk phosphatidylcholine (Lipid Products) essentially according to the method of Keana et al. (1986). All other spin-labels were synthesized according to procedures detailed in Marsh and Watts (1982).

Sample Preparation. Typically $1 \mathrm{mg}$ of lipid or lipid mixture containing $1 \mathrm{~mol} \%$ spin-label was taken to dryness from a chloroform solution to yield a thin film at the bottom of a glass vial. The thin film was hydrated by adding $25 \mu \mathrm{L}$ of buffer ( $10 \mathrm{mM}$ Tris, $10 \mathrm{mM} \mathrm{NaCl}, 0.1 \mathrm{mM}$ EDTA, pH $8.0)$ and dispersed by vortexing. For the pH-dependence experiments, appropriate buffer salts were used to prepare 10 $\mathrm{mM}$ buffer $/ 10 \mathrm{mM} \mathrm{NaCl} / 0.1 \mathrm{mM}$ EDTA solutions. Lipidprotein complexes were prepared by adding an excess $(2.5 \mathrm{mg})$ of MBP in the same buffer from a stock solution of $1 \mathrm{mg} / \mathrm{mL}$. The complexes, which precipitated instantaneously, were pelleted in a bench centrifuge and transferred to $100-\mu \mathrm{L}$ glass capillaries that were used for ESR measurements. The pellets were dissolved, after the ESR experiments, by adding a few

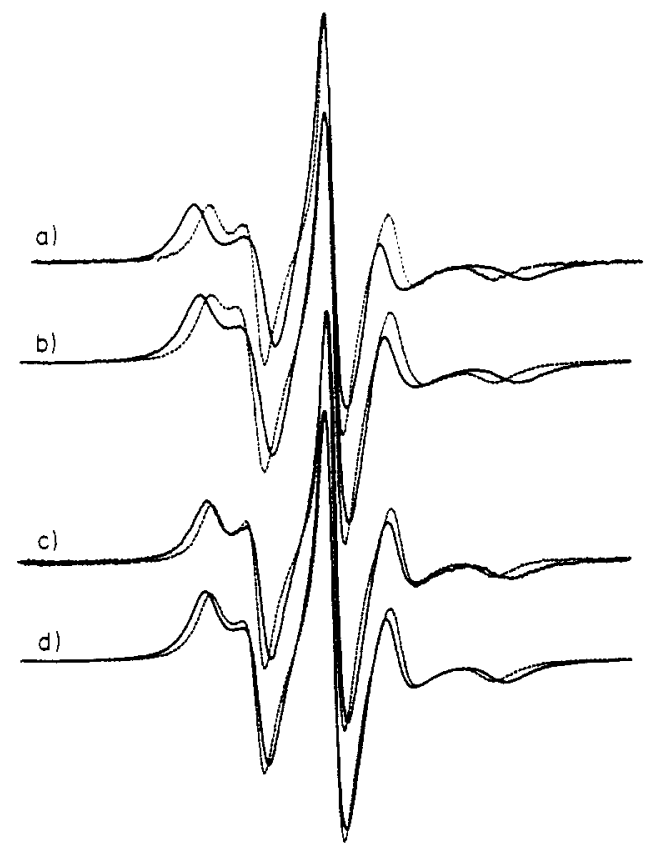

FIGURE 1: ESR spectra at $40^{\circ} \mathrm{C}$ of the 5-PGSL and 5-PCSL phospholipid spin-labels in mixed bilayers of DMPG and DMPC and in DMPG/DMPC complexes with saturating amounts of MBP. Buffer: $10 \mathrm{mM}$ Tris, $10 \mathrm{mM} \mathrm{NaCl}$, and $0.1 \mathrm{mM}$ EDTA, pH 8.0. The solid line of each pair is the spectrum from the protein complexes, and the dotted line is the spectrum from the mixed bilayers. (a) 5-PGSL in MBP-DMPG complexes $\left(X_{\mathrm{DMPC}}=0\right)$; (b) 5-PCSL in MBP-DMPG complexes $\left(X_{\mathrm{DMPC}}=0\right)$; (c) 5-PGSL in MBP(DMPG/DMPC) complexes $\left(X_{D M P C}=0.5\right)$; (d) 5 -PCSL in MBP-(DMPG/DMPC) complexes $\left(X_{\text {DMPC }}=0.5\right)$. The total scan width was $100 \mathrm{G}$.

drops of $1 \mathrm{~N} \mathrm{NaOH}$ and analyzed for lipid phosphate and protein contents according to procedures described by Eibl and Lands (1969) and Lowry et al. (1951), respectively.

ESR Spectroscopy. ESR experiments were performed on a Varian E-12 Series 9-GHz spectrometer interfaced to a PDP 11/10 computer system. Sample temperatures were controlled by a nitrogen gas flow regulation system and were measured by using a fine-wire thermocouple that was placed close to the sample at the top of the microwave cavity. Spectral subtractions were performed as described earlier (Marsh, 1982; Sankaram et al., 1989). The outer hyperfine splitting, $2 A_{\max }$, was used to characterize the spectra of the C-5 spin-labels. Comprehensive line-shape simulations of the ESR spectra from such spin-labels in fluid-phase bilayers have shown that there are important contributions from slow molecular motions (Lange et al., 1985). Thus, the outer hyperfine splittings are sensitive to both the amplitude and rate of the lipid chain motions and can be used to characterize the strength of interaction of the various spin-labeled lipids with the protein.

\section{RESULTS}

Lipid Mixtures. A systematic investigation of the protein binding and of the accompanying changes in the spectral parameters for the 5- and 12-position-labeled PGSL and PCSL in DMPG/DMPC lipid mixtures was carried out. The selectivity of interaction of MBP with phosphatidylglycerol relative to phosphatidylcholine is reflected in the ESR spectra of the 5-PGSL and 5-PCSL spin-labels in MBP-(DMPG/ DMPC) complexes (see Figure 1). In complexes of MBP with DMPG/DMPC mixtures, the ESR spectra of both the 5PGSL and 5-PCSL spin-labels have larger outer hyperfine splittings than in mixtures of the lipids alone, indicating a reduction of the acyl chain mobility of both lipid species on binding of the protein. At the lower mole fractions of DMPC 


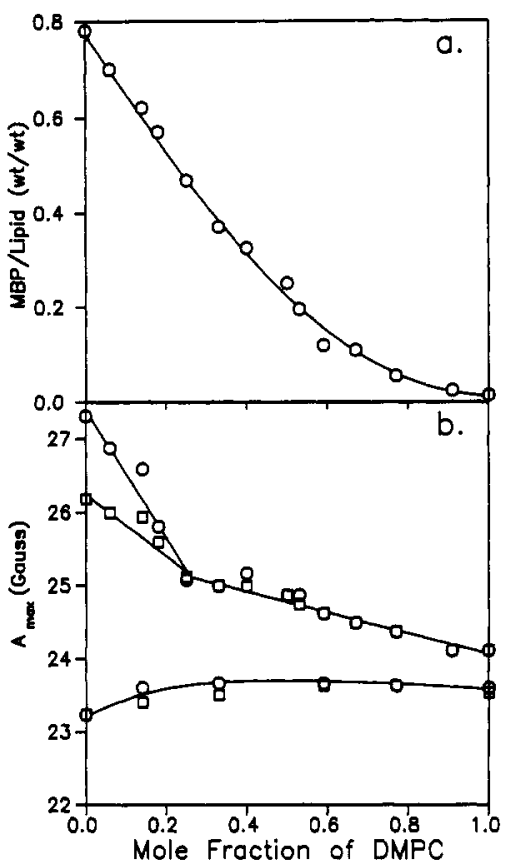

FIGURE 2: Dependence of the protein binding and spin-label outer hyperfine splitting $\left(2 A_{\max }\right)$ at saturation on the mole fraction of DMPC $\left(X_{\mathrm{DMPC}}\right.$ ) in DMPG/DMPC mixed bilayers. (a) MBP binding (for $2.5: 1 \mathrm{w} / \mathrm{w}$ protein/lipid added); (b) $A_{\max }$ at $40^{\circ} \mathrm{C}$ for 5-PGSL (O) and 5-PCSL ( $\square$ ) in the protein-lipid complexes (upper curves) and in the mixed lipid bilayers (lower curve). Buffer: $10 \mathrm{mM}$ Tris, 10 $\mathrm{mM} \mathrm{NaCl}$, and $0.1 \mathrm{mM}$ EDTA, $\mathrm{pH}$ 8.0.

$\left(X_{\mathrm{DMPC}}\right)$, the increase in hyperfine splitting is greater for the 5-PGSL spin-label than for the 5-PCSL spin-label, whereas at the higher mole fractions of the zwitterionic lipid the degrees of spectral perturbation on binding of the protein become much more comparable. In addition, the overall spectral perturbations induced by the protein become smaller for both labels as the mole fraction of DMPC increases.

The variation in the values of the outer hyperfine splitting, $2 A_{\max }$, of the C-5 spin-labels as a function of the mole fraction of DMPC, $X_{\mathrm{DMPC}}$, is shown in Figure 2b. The saturation binding values of MBP to the DMPG/DMPC mixed bilayers are also given in Figure 2a. Consistent with earlier observations (Boggs \& Moscarello, 1978b; Papahadjopoulos et al., 1975; Sankaram et al., 1989), it is seen from Figure 2a that the MBP binds strongly to DMPG bilayers with a lipid/protein ratio of $36: 1 \mathrm{~mol} / \mathrm{mol}$ at saturation but does not bind appreciably to bilayers of DMPC alone. The dependence of the binding on mole fraction of DMPC, $X_{\mathrm{DMPC}}$, is not linear, indicating that the mode of protein binding to DMPG in the mixed bilayers is different from the binding to pure DMPG bilayers. The values of the outer hyperfine splitting, $2 A_{\max }$, and the spectral line shapes of both 5-PGSL and 5-PCSL in mixtures of the lipids alone are very similar (lower curve in Figure $2 b$ ). This indicates that the two spin-labels interact similarly with both DMPG and DMPC in the bilayer, as might be expected from the near ideal mixing of these two lipids (Sixl \& Watts, 1982). However, protein binding has a differential effect on the ESR spectra of the 5-PGSL and 5-PCSL spinlabels, indicating a different degree of interaction with these two lipids (upper curve in Figure 2b). A selective interaction with the 5-PGSL label is observed at mole fractions of DMPC up to $X_{\mathrm{DMPC}}=0.25$. Beyond this mole fraction the values of the outer hyperfine splittings of the two labels are still considerably greater in the lipid-protein complexes than in the pure mixed lipid bilayers, reflecting the significant degree of protein binding (cf. Figure 2a), but differences between the spectra of 5-PGSL and 5-PCSL are no longer discernible in

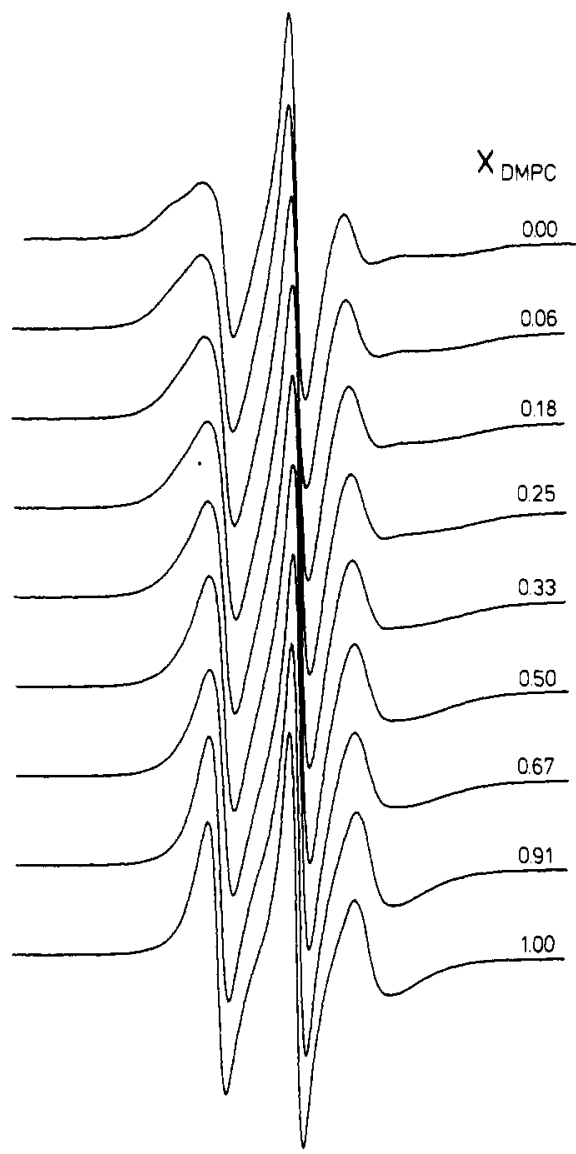

FIGURE 3: ESR spectra of the 12-PGSL spin-label in MBP(DMPG/DMPC) complexes at $30^{\circ} \mathrm{C}$ for different mole fractions of DMPC, $X_{\mathrm{DMPC}}$. The values of $X_{\mathrm{DMPC}}$ are indicated in the figure. Buffer: $10 \mathrm{mM}$ Tris, $10 \mathrm{mM} \mathrm{NaCl}$, and $0.1 \mathrm{mM}$ EDTA, pH 8.0 . The total scan width was $100 \mathrm{G}$.

the lipid-protein complexes. At these higher mole fractions of DMPC, the interaction of the MBP with the lipids is much more homogeneous in nature than at mole fractions less than 0.25 .

Two-Component Spectra. The ESR spectra of the 12PGSL and 12-PCSL spin-labels in MBP complexes with the mixed lipid system are two component in nature at low mole fractions of DMPC. The spectra of the 12-PGSL spin-label in lipid-protein complexes with mole fractions of DMPC varying over the entire range from $X_{\mathrm{DMPC}}=0$ to $X_{\mathrm{DMPC}}=1$ are given in Figure 3. Two components are clearly seen in the spectra until a mole fraction of $X_{\mathrm{DMPC}}=0.25$. The component seen in the outer wings of the spectra is motionally restricted by the protein and represents the fraction of lipids directly in contact with it. The other component corresponds to fluid lipids that are in slow exchange with the protein-associated lipids on the conventional nitroxide ESR time scale and have a spectral line shape very similar to that of the lipids alone. The slow exchange leads only to a slight broadening of this fluid component. The two-component nature of the spectra was substantiated and quantitated as described earlier (Marsh, 1982; Görrissen et al., 1986; Sankaram et al., 1989). Briefly, the method involves subtracting a fluid lipid spectrum obtained for the spin-label in pure lipid bilayers from the spectrum of the lipid-protein recombinant. The difference spectrum represents the motionally restricted spin-labeled lipid component. Alternatively, a difference spectrum may be obtained by intersubtractions between a pair of spectra from lipid-protein complexes that contain different proportions of the two spectral components [for a description of intersubtractions see Knowles et al. (1981)]. The difference spectra 


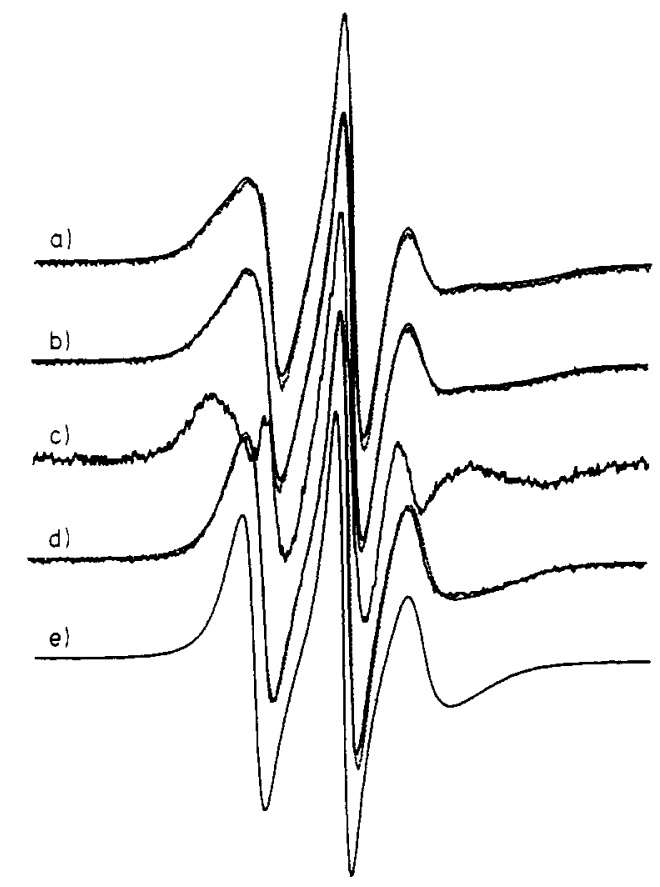

FIGURE 4: Quantitation of the motionally restricted spectral component from lipid-protein complexes. ESR spectra of 12-PGSL in (a) $\mathrm{MBP}-(\mathrm{DMPG} / \mathrm{DMPC})$ complex $\left(X_{\mathrm{DMPC}}=0.06\right.$ at $\left.30^{\circ} \mathrm{C}\right) ;(\mathrm{b})$ MBP-(DMPG/DMPC) complex $\left(X_{\mathrm{DMPC}}=0.14\right.$ at $\left.30^{\circ} \mathrm{C}\right) ;(\mathrm{c})$ difference spectrum obtained by subtracting spectrum $b$ from spectrum a; (d) difference spectrum obtained by subtracting spectrum $a$ from spectrum $b$ (dotted line represents DMPG bilayers at $24^{\circ} \mathrm{C}$ ); (e) DMPG bilayers at $30^{\circ} \mathrm{C}$. Dotted lines in (a) and (b) are additions of spectrum $\mathrm{c}$ and spectrum $\mathrm{d}$ in relative proportions consistent with the subtractions. The total scan width was $100 \mathrm{G}$.

obtained by the two methods may then be compared.

The results of the quantitative analysis of the two-component spectra are shown in Figure 4. For the intersubtractions, the ESR spectra at $30^{\circ} \mathrm{C}$ in lipid-protein complexes with different mole fractions of DMPC (Figure 4a,b) were used. As mentioned above, these spectra are two component in nature, as opposed to the ESR spectrum of 12-PGSL in pure lipid bilayers at $30^{\circ} \mathrm{C}$ (Figure 4e), which consists solely of a single component. Subtracting the two-component spectrum with higher mole fraction of DMPC (Figure 4b) from that with lower fraction of DMPC (Figure 4a) yields a motionally restricted component as the difference spectrum, which is shown in Figure 4c. When performed in the reverse direction, the intersubtractions yield a difference spectrum (Figure 4d) that represents the fluid lipid population and closely resembles the spectrum of the 12-PGSL spin-label in pure lipid bilayers at $24^{\circ} \mathrm{C}$ (Figure $4 d$, dotted line). Similar intersubtractions were performed with spectra of the 12-PCSL label in lipid-protein complexes with different mole fractions of DMPC. The intersubtraction strategy was further validated by performing spectral additions of the single-component difference spectra (Figure $4 c, d$ ), using the relative proportions of the two components that were calculated from the subtraction factors obtained from the complementary intersubtractions. These addition spectra are given by the dotted lines in Figure 4a,b and agree very closely with the original two-component spectra.

The results of the spectral subtractions are given in Table I. The motionally restricted fraction of the 12-PGSL spinlabel is consistently higher than that of the 12-PCSL spin-label at all mole fractions of DMPC for which a two-component spectrum is seen from the mixed lipid-protein complexes. This parallels the increased outer hyperfine splitting seen in the selectivity of the 5-PGSL spin-label relative to the 5-PCSL reported above. For both $\mathrm{C}-12$ labels, the fraction of lipid that
Table I: Fractions of Motionally Restricted 12-PGSL and 12-PCSL Spin-Labels in MBP-(DMPG/DMPC) Complexes as a Function of Mole Fraction of DMPC

\begin{tabular}{ccc} 
& \multicolumn{2}{c}{ fraction restricted } \\
\cline { 2 - 3 }$X_{\text {DMPC }}$ & 12-PGSL & 12-PCSL \\
\hline 0.0 & 0.50 & 0.30 \\
0.06 & 0.43 & 0.26 \\
0.18 & 0.34 & 0.15 \\
0.25 & 0.32 & 0.11 \\
0.33 & 0.21 & \\
\hline
\end{tabular}

is motionally restricted decreases with increasing mole fraction of DMPC in the lipid-protein complexes and in this way correlates with the decrease in the amount of protein bound (cf. Figure 2a).

Phase Transition Behavior. Aqueous dispersions of both DMPC and DMPG alone exhibit a gel to liquid-crystalline phase transition at approximately $23^{\circ} \mathrm{C}$. Mixtures of these two lipids in the mole fraction range $0-1$ also melt at temperatures close to this, without any appreciable broadening of the transition width. The effect of protein binding on this phase transition was studied by monitoring the central line height of the ESR spectra of the 5-PGSL spin-label while the temperature was scanned continuously [for a description of the method see Watts et al. (1978)]. In the presence of a saturating amount of protein, a phase transition is still observed for mole fractions of DMPC greater than 0.6. At mole fractions of DMPG greater than 0.4, the phase transition is completely abolished by the protein binding. In the composition range for which a transition is still observed in the presence of protein, the transition temperature increases slightly, with accompanying broadening of the transition width, as the DMPG content increases. The maximum temperature that the mid-point of the transition reaches is $27^{\circ} \mathrm{C}$, and the upper transition boundary extends to no higher than $35^{\circ} \mathrm{C}$. The values of $A_{\max }$ measured for the 5-PGSL spin-label at 40 ${ }^{\circ} \mathrm{C}$ (Figure 2b), and the spectra given in Figure 1, therefore correspond to the fluid liquid-crystalline state of the lipidprotein complexes at all lipid compositions. In addition, the analysis of the two-component spectra for the 12-position labels at $30^{\circ} \mathrm{C}$ was performed only for samples with $X_{\mathrm{DMPC}} \leq 0.25$, for which the phase transition is completely abolished and the spectra can be assumed to correspond to the liquid-crystalline phase.

Phospholipid Selectivity. ESR spectra of selected diacyl lipids spin-labeled at the fifth position in the $s n-2$ chain and incorporated into DMPG-MBP complexes are given in Figure 5. Included are spectra of spin-labeled phosphatidylserine (5-PSSL), phosphatidylinositol (5-PISL), phosphatidylethanolamine (5-PESL), and diacylglycerol (5-DGSL), at 30 ${ }^{\circ} \mathrm{C}$, both in the presence and in the absence of saturating amounts of MBP. The spectra of all labels display a larger outer hyperfine splitting in the presence of protein than in the absence of protein, indicating a reduction in mobility of the acyl chains of all the different lipids on binding of the protein. The extent of this motional perturbation displays a very clear dependence on the lipid species, being greatest for phosphatidylserine and smallest for diacylglycerol.

The values for the outer hyperfine splittings, $2 A_{\max }$, for eight different C-5 spin-labels, both in pure DMPG bilayers and in MBP-DMPG complexes at $30^{\circ} \mathrm{C}$, are given in Table II. The spectra of all the various diacylphospholipid spin-labels in pure DMPG bilayers are very similar (only that of diacylglycerol has an appreciably smaller spectral anisotropy): the values for the outer hyperfine splittings at $30^{\circ} \mathrm{C}$ are nearly identical $\left(A_{\max }=25.2 \pm 0.2 \mathrm{G}\right)$. This indicates that, with the 


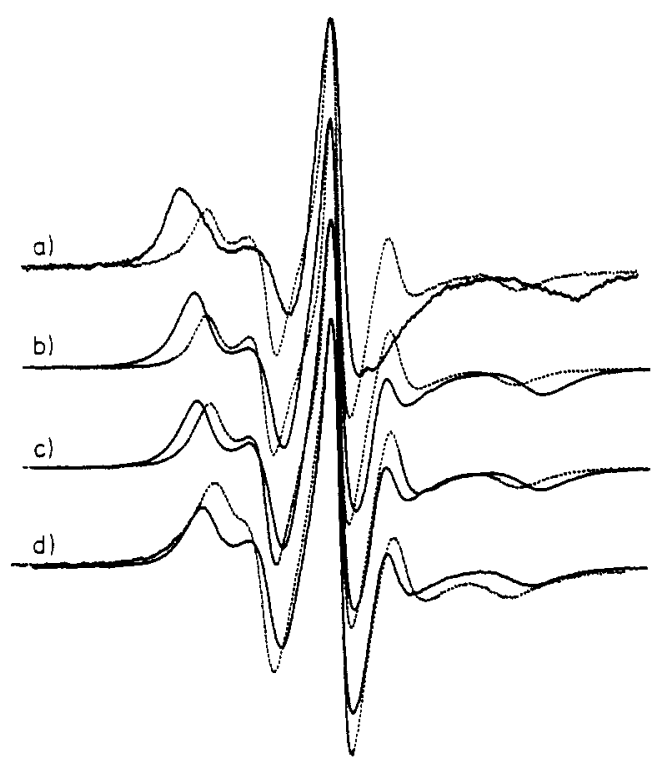

FIGURE 5: ESR spectra at $30^{\circ} \mathrm{C}$ of phospholipid spin-labels labeled at the fifth position of the $s n-2$ chain in DMPG bilayers and in DMPG-MBP complexes ( $0.77 \mathrm{mg}$ of MBP bound $/ \mathrm{mg}$ of DMPG). Buffer: $10 \mathrm{mM}$ Tris, $10 \mathrm{mM} \mathrm{NaCl}$, and $0.1 \mathrm{mM}$ EDTA, pH 8.0 . The solid line of each pair is the spectrum from the lipid-protein complex, and the dotted line is the spectrum from DMPG bilayers. (a) Phosphatidylserine spin-label (5-PSSL); (b) phosphatidylinositol spin-label (5-PISL); (c) phosphatidylethanolamine spin-label (5. PESL); (d) diacylglycerol spin label (5-DGSL). The total scan width was $100 \mathrm{G}$.

Table II: Outer Hyperfine Splittings $\left(2 A_{\max }\right)$ at $30^{\circ} \mathrm{C}$ of Phospholipid Spin-Labels Labeled at the Fifth Position of the $s n-2$ Chain Incorporated in DMPG Bilayers and DMPG-MBP Complexes $^{a}$

\begin{tabular}{lcccc}
\hline & & DMPG & DMPG + MBP & \\
spin-label & charge & $A_{\max }(\mathrm{G})$ & $A_{\max }(\mathrm{G})$ & $\Delta A_{\max }(\mathrm{G})$ \\
\hline 5-PSSL & - & 25.36 & 32.33 & 6.97 \\
5-CLSL & - & 24.67 & 28.94 & 4.27 \\
5-PASL & $2-$ & 25.24 & 29.26 & 4.02 \\
5-PGSL & - & 25.05 & 28.44 & 3.39 \\
5-PISL & - & 25.03 & 28.36 & 3.33 \\
5-PASL & - & 24.96 & 28.23 & 3.27 \\
5-PESL & \pm & 25.05 & 28.23 & 3.18 \\
5-PCSL & \pm & 25.05 & 28.00 & 2.95 \\
5-DGSL & 0 & 24.17 & 27.00 & 2.83 \\
\hline
\end{tabular}

${ }^{\circ} \Delta A_{\max }$ is the increase in outer hyperfine splitting constant induced on binding of the protein. ${ }^{b} \mathrm{pH} 10.1$.

exception of 5-DGSL, all spin-labels interact similarly with the host lipid and have the same vertical location in the bilayer. Complexation of the MBP to DMPG bilayers has a large broadening effect on the spectral shapes of the incorporated spin-labels. Thus, the difference in the values of $A_{\max }$ between the lipid-protein complexes and the pure lipid can be taken as a measure of the degree of perturbation of the lipid chain motion and hence of the selectivity of interaction of the lipid with the protein. This is especially useful in the comparison with 5-DGSL (and to a lesser extent 5-CLSL), which has a smaller value of $A_{\max }$ in the pure lipid. These values of $\Delta A_{\max }$ are given in the final column of Table II. A differential selectivity in the interaction with MBP is seen clearly from the values of $A_{\max }$. Relative to the 5-PGSL spin-label in DMPG bilayers, the singly negatively charged lipids 5-CLSL and 5-PSSL and the doubly negatively charged 5-PASL exhibit greater increases in $A_{\max }$, whereas the protein-induced increase in $A_{\max }$ is less for the other two singly negatively charged lipid spin-labels, 5-PISL and 5-PASL. The zwitterionic lipids, 5-PCSL and 5-PESL, show the least selectivity of interaction with MBP among the phospholipid spin-labels.

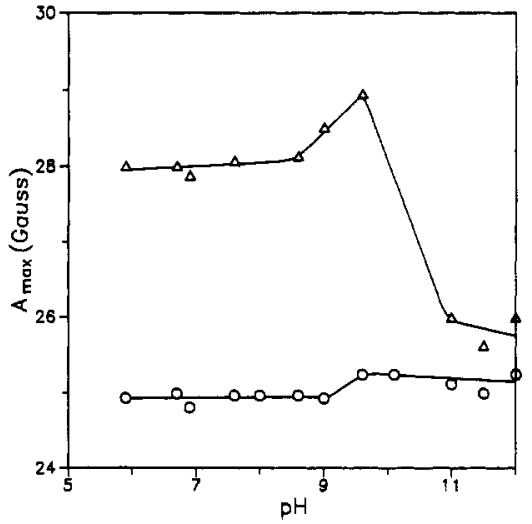

FIGURE 6: $\mathrm{pH}$ titration of the outer hyperfine splittings, $2 A_{\max }$, of the 5-PASL phosphatidic acid spin-label in DMPG bilayers $(O)$ and in DMPG-MBP complexes $(\Delta)$ at saturation protein binding. Measurement temperature was $30^{\circ} \mathrm{C} ; 10 \mathrm{mM}$ buffers containing 10 $\mathrm{mM} \mathrm{NaCl}$ and $0.1 \mathrm{mM}$ EDTA were used.

The neutral lipid spin-label, 5-DGSL, has an intrinsically lower value of $A_{\max }$, suggesting that it is located somewhat deeper in the bilayer. This label also exhibits the least, although still significant, increase in $A_{\max }$ on binding of the protein.

$p H$ Titration of Phosphatidic Acid. Further insight into the contribution of the phospholipid headgroup to the selectivity of interaction with MBP is obtained from $\mathrm{pH}$ titration with the 5-PASL spin-label. When incorporated in the membrane, this phospholipid has a $\mathrm{p} K_{\mathrm{a}}$ that lies within an easily accessible range [cf. Horvăth et al. (1988)]. The dependence of the values $A_{\max }$ at $30^{\circ} \mathrm{C}$ on pH for 5-PASL in pure DMPG dispersions and in MBP-DMPG complexes is given in Figure 6. The data in negatively charged DMPG bilayers alone yield an interfacial $\mathrm{p} K_{\mathrm{a}}$ of 9.3 for the $\mathrm{PA}^{-}$to $\mathrm{PA}^{2-}$ titration, as seen by the small increase in $A_{\max }$ on increasing the $\mathrm{pH}$ through this value. The value of $\mathrm{p} K_{\mathrm{a}}$ for this titration is not significantly changed by binding of the MBP. The titration of 5-PASL in the DMPG-MBP complexes ( $\mathrm{p} K_{\mathrm{a}}$ $=9.3$ ) overlaps with the titration of the basic amino acid side chains of MBP $\left(\mathrm{p} K_{\mathrm{a}}=10.6\right)$. The protein titration has been shown previously to be accompanied by a parallel decrease in binding of MBP to DMPG bilayers (Sankaram et al., 1989). This is seen here by the large decrease in the value of $A_{\max }$ for the lipid-protein complexes at high $\mathrm{pH}$ to levels comparable to those found with the lipid alone. Despite this overlap between the titrations of the lipid spin-label and of the protein, it is seen that the increase in $A_{\max }$ at the $\mathrm{p} K$ of the 5-PASL spin-label is considerably greater in the presence than in the absence of protein. Hence, the selectivity is greater for the lipid in the doubly negatively charged state than it is in the singly charged state.

\section{Discussion}

Lipid Mixtures. A differential dependence of the outer hyperfine splittings of the 5-PGSL and 5-PCSL spin-labels on the mole fraction of DMPC is clearly seen in Figure 2. The preferential interaction of phosphatidylglycerol relative to phosphatidylcholine is seen until a mole fraction of DMPC of 0.25 , beyond which the difference in selectivity disappears despite significant levels of protein binding (Figure 2). This result strongly suggests that the mode of interaction with the mixed lipid bilayers is different at the higher mole fractions of the zwitterionic lipid than it is at the higher mole fractions of the negatively charged component. Apart from this difference, two principal factors affect the dependence on bilayer composition of the degree of association of the two lipids with the protein. The first factor is the decreasing extent of protein 
binding with increasing mole fraction of DMPC (Figure 2a), which will decrease the motional perturbation of both lipid species in the same fashion. The second factor is the competition of the two spin-labeled lipids with the background host lipid of changing composition. As the mole fraction of DMPC increases, the competition of both spin-labeled lipids should be more effective, since the proportion of DMPG, which has the higher selectivity, is decreasing. The analysis of the selectivity given below suggests that this latter effect would preserve a preferential association of phosphatidylglycerol relative to phosphatidylcholine for all mole fractions of DMPC. This further supports the suggestion that the mode of MBP association is different at high mole fractions of DMPC. It is interesting to note that a preferential interaction of MBP with the phosphatidylglycerol component has been found with equimolar mixtures of phosphatidylcholines and phosphatidylglycerols of different chain compositions (Boggs et al., 1977). In this case, the difference in chain composition may serve to extend the composition range over which the preferential interaction is observed.

The dependence of the outer hyperfine splitting, $A_{\max }$, of the C-5 labels on lipid selectivity may be analyzed quantitatively if it is assumed that the ESR spectra correspond to fast exchange on the conventional nitroxide ESR time scale [see, e.g., Carrington and McLachlan (1969)]. ${ }^{2}$ The model assumes that there are a limited number, $n_{p}$, of relatively specific association sites on the protein at which the spin-labeled lipids have a maximum hyperfine splitting constant, $A^{\mathrm{p}}$, and the remainder of the lipids have a lower hyperfine splitting constant, $A^{\mathrm{f}}$, which cannot be less than that in the pure lipid. The criterion for fast exchange is that the rate of exchange between the "free" and protein-associated lipid components shall be greater than the difference between the hyperfine splittings of these two components. The maximum value of $A_{\max }$ in Table II (that corresponding to 5-PSSL) can be taken as an approximate upper limit and has a value of $\approx 2 \times 10^{7} \mathrm{~s}^{-1}$. Typical lipid translational diffusion rates in fluid DMPC bilayers are $\nu_{\text {dif }}=4 D_{\Upsilon} /\left\langle x^{2}\right\rangle \approx 7-9 \times 10^{7} \mathrm{~s}^{-1}$ (Sachse et al., 1987), and our unpublished values for DMPG in the presence of MBP are slower but of a similar order of magnitude. Thus, the assumption of fast exchange may hold, at least to a first approximation.

With the above model, the outer hyperfine splitting constant is then given by the usual expression for fast exchange

$$
A_{\max }=\left(A^{\mathrm{p}}-A^{\mathrm{f}}\right) f+A^{\mathrm{f}}
$$

where $f$ is the fraction of the spin-labeled lipids that is associated with the specific sites (assumed to be totally occupied by either labeled or unlabeled lipids). For simplicity, it is further assumed that the spin-labeled lipids directly reflect the selectivity of the corresponding unlabeled lipids in the DMPG/DMPC mixtures, in a 1:1 fashion. The fraction, $f$, of the lipid component with mole fraction $X$ that occupies specific sites is then related to the extent of protein binding,

\footnotetext{
2 An alternative explanation for the differences in $A_{\max }$ between the various lipid labels which should be considered is that the different lipids have the same extent of binding but display intrinsically different degrees of motional restriction when bound to the protein. However, it is difficult to explain the dependence of $A_{\max }$ on DMPG/DMPC ratio (Figure 2) with this model. If such effects are present, it seems that they are outweighed by the differences in extents of binding, which are better described with the fast exchange model. For this reason, the latter model is preferred. It should also be mentioned that attempts to decompose the spectra of Figures 1 and 5 by spectral subtraction using the spectra from the pure lipid were unsuccessful, suggesting that they do not consist of two unresolved components in slow exchange.
}

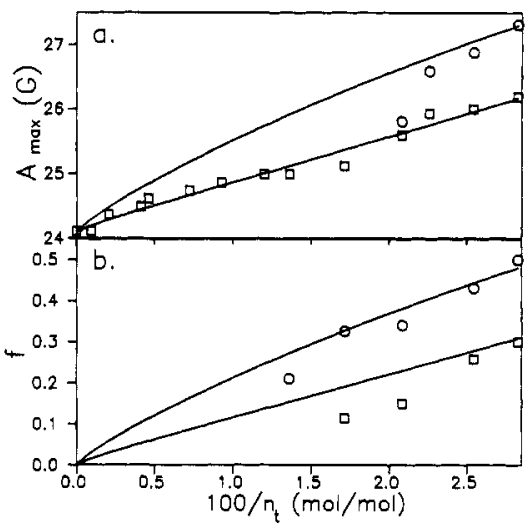

FIGURE 7: (a) Dependence of the outer hyperfine splitting constant, $A_{\text {max }}$, on the mole ratio of MBP/lipid bound, $1 / n_{t}$, in DMPG/DMPC mixtures. ( $\square$ ) Experimental data for 5-PCSL; (O) data for 5-PGSL. The data for 5-PGSL for $X_{\mathrm{DMPC}} \geq 0.25$ have been omitted for clarity, since they are indistinguishable from those for 5-PCSL. The lower curve represents a least-squares fit to the data for 5-PCSL according to eq 1 and 2, yielding the optimized parameters $n_{\mathrm{p}}=17, K_{\mathrm{r}}(\mathrm{PC}: \mathrm{PG})$ $=0.484, A^{\mathrm{P}}=30.77 \mathrm{G}$, and $A^{\mathrm{f}}=24.09 \mathrm{G}$. The upper curve represents the corresponding dependence predicted for 5-PGSL, by use of the same parameters, i.e., with $K_{\mathrm{r}}(\mathrm{PG}: \mathrm{PC})=1 / K_{\mathrm{r}}(\mathrm{PC}: \mathrm{PG})=2.07$. (b) Dependence of the fraction of motionally restricted spin-label, $f$, on the mole ratio of MBP/lipid bound, $1 / n_{t}$, in DMPG/DMPC mixtures. (D) Experimental data for 12-PCSL; $(0)$ data for 12-PGSL. The curves are the predicted dependences according to eq 2 by use of the parameters $n_{\mathrm{p}}=17$ and $K_{\mathrm{r}}(\mathrm{PC}: \mathrm{PG})=1 / K_{\mathrm{r}}(\mathrm{PG}: \mathrm{PC})=0.484$, from the least-squares fit in (a).

$1 / n_{t}$, via the equation for equilibrium lipid-protein association [see, e.g., Brotherus et al. (1981) and Marsh (1985)]

$$
\left.1 / n_{\mathrm{t}}=f X+(1-X) /\left[f+(1-f) K_{\mathrm{r}}\right]\right\} / n_{\mathrm{p}}
$$

where $K_{\mathrm{r}}$ is the association constant of the lipid component of mole fraction $X$ relative to that of the second lipid component and $n_{\mathrm{t}}$ is the total lipid/protein mole ratio. ${ }^{3}$ The dependence of the values of $A_{\max }$ for 5-PCSL on the extent of protein binding, $1 / n_{\mathrm{t}}$, for MBP-(DMPG/DMPC) complexes of different lipid composition is given in Figure 7a. A nonlinear least-squares fit to the data, based on eq 1 and 2 , yields values of $n_{\mathrm{p}}=17$ and $K_{\mathrm{r}}(\mathrm{PC}: \mathrm{PG})=0.484$. The fit is reasonably satisfactory, except for the data point at $X_{D M P C}$ $=0.25$. However, it is not possible to obtain a consistent fit to the data for the 5-PGSL label by using the same parameters. With $n_{\mathrm{p}}=17$ and $K_{\mathrm{r}}(\mathrm{PG}: \mathrm{PC})=1 / K_{\mathrm{r}}(\mathrm{PC}: \mathrm{PG})=2.07$, it is possible to account for the data from 5-PGSL in a MBPDMPG complex in the absence of DMPC, but for increasing mole fractions of DMPC, the values of $A_{\max }$ are predicted always to remain greater than those for 5-PCSL. In contrast, the experimental hyperfine splittings for 5-PGSL become almost the same as those for 5-PCSL at mole fractions of DMPC greater than 0.25 (cf. Figure $2 \mathrm{~b}$ ). This result confirms that the dependence of the selectivity on the content of zwitterionic lipid cannot be explained simply on the basis of a single mode of lipid-protein association at all mole fractions.

Hydrophobic Lipid-Protein Association. The two-component spectra observed for the lipid-protein complexes labeled with either 12-PGSL (Figure 3) or 12-PCSL (data not shown) provide evidence for the existence of two classes of lipids of different mobility in the MBP-(DMPG/DMPC) complexes at mole fractions of DMPC below 0.25 . These two components correspond to protein-associated and fluid lipids whose different rates of acyl chain motion make them resolvable on the con-

\footnotetext{
${ }^{3}$ For protein binding to DMPG alone $(X=1)$, eq 1 and 2 predict that $A_{\text {max }}$ should be linearly dependent on the protein to lipid ratio, $1 / n_{\text {. }}$. This is found to be the case for values of $1 / n_{\mathrm{t}}$ less than saturation binding (data not shown), which further supports the fast exchange model.
} 
ventional ESR time scale. The difference in hyperfine splittings of the fluid and motionally restricted components is approximately twice the maximum value of $\Delta A_{\max }$ observed for the 5-position labels, consistent with one set of spectra approximately fulfilling the slow exchange and the other the fast exchange condition, respectively. It would seem that the spectra of the 5-position and 12-position labels lie very close to (but on opposite sides of) the critical exchange criterion. The spectra of the two components (cf Figure 4) indicate that the extent of protein-induced motional restriction is similar for the 12-PGSL and 12-PCSL labels. The relative proportions of the motionally restricted and fluid lipids are different for the two labels, reflecting the selectivity of interaction.

The effects of the lipid composition on the proportion of motionally restricted component for the C-12 labels can, in principle, be predicted by using eq 2 for the equilibrium lipid-protein association in the lipid mixtures (cf. above). The dependence of the fraction, $f$, of motionally restricted 12-PGSL and 12-PCSL on the extent of protein binding, $1 / n_{t}$, for the different lipid mixtures is given in Figure $7 \mathrm{~b}$. With the parameters $n_{\mathrm{p}}=17$ and $K_{\mathrm{r}}(\mathrm{PC}: \mathrm{PG})=1 / K_{\mathrm{r}}(\mathrm{PG}: \mathrm{PC})=0.484$, obtained from the fit of the 5-PCSL data in Figure 7a, eq 2 successfully predicts the relative selectivities of 12-PGSL and 12-PCSL in MBP complexes with DMPG alone. This suggests that there is a close relationship between the postulated specific protein sites $\left(n_{\mathrm{p}}\right)$ giving rise to the increase in $A_{\max }$ for the 5-position labels and those giving rise to the direct motional restriction of the chains of the 12-position lipid labels. At the lower mole fractions of DMPC, the model gives a better description of the dependence of the interaction on DMPC content of the lipid mixture for the 12-PGSL label than was predicted above for the 5-PGSL label. However, the fit for the 12-PCSL label is not so good. Deviations from the measured values for the latter might be accounted for partly by the technical difficulties in resolution of the two components for low values of $f$. This difficulty also hampers an accurate determination of the lipid composition at which the motionally restricted component is no longer present. However, the model definitely predicts larger values of $f$ than are observed at the high DMPC contents (Figure 7b).

The shortcomings of the above model may be due to a varying stoichiometry of the lipid-protein interaction with lipid composition. The disappearance of the differences between the PGSL and PCSL labels at mole fractions of DMPC greater than 0.25 suggests that until this value the relative distribution of charged and zwitterionic lipids satisfies the stoichiometry required by the basic amino acid residues of the MBP. Beyond this mole fraction, a conformational change of the protein is possibly required to match the lipid distribution in the bilayer at higher concentrations of DMPC. Such a conformational change possibly involves a disruption of any folded structures capable of penetrating the lipid bilayer.

The binding stoichiometry of $36: 1$ observed for the DMPG-MBP complexes has been shown to be in agreement with a prolate ellipsoid structure for the protein with dimensions of $150 \times 15 \AA$ (Epand et al., 1974; MacNaughtan et al., 1985; Sankaram et al., 1989). Dimensions of the unfolded structure that the protein might assume in the lipid mixtures with high DMPC content could be estimated from the intrinsic viscosity of the denatured MBP in aqueous $6 \mathrm{M}$ guanidine hydrochloride solutions (Epand et al., 1974). The axial ratio of a prolate ellipsoid structure thus calculated would be 17:1, which is consistent with approximate dimensions of $229 \times 13.5$ $\AA$. For complete coverage of the negatively charged membrane surface, a lipid/protein ratio of 50:1 is calculated by assuming
Table III: Relative Association Constants, $K_{\mathrm{r}}$, for C-5 Spin-Labeled Lipids with MBP in DMPG Bilayers, Calculated by Assuming Fast Exchange, According to Equation 4

\begin{tabular}{cccccc}
\hline spin-label & charge & $K_{\mathrm{r}}$ & spin-label & charge & $K_{\mathrm{r}}$ \\
\hline 5-PSSL & - & 37.1 & 5-PASL & - & 0.9 \\
5-CLSL & - & 1.6 & 5-PESL & \pm & 0.9 \\
5-PASL & $2-$ & 1.4 & 5-PCSL & \pm & 0.8 \\
5-PGSL & - & 1.0 & 5-DGSL & 0 & 0.7 \\
5-PISL & - & 1.0 & & & \\
\hline
\end{tabular}

an area of $60 \AA^{2}$ per lipid molecule. The DMPG/MBP ratios calculated from the experimentally determined (DMPG + DMPC)/MBP ratios in the range $1.0>X_{\mathrm{DMPC}}>0.25$ are between 51 and 127, in agreement with an unfolding of the protein conformation with increasing mole fraction of DMPC.

Lipid Selectivity. The selectivity of interaction of the myelin basic protein with negatively charged and zwitterionic phospholipids and diacylglycerol was studied. The outer hyperfine splittings, $2 A_{\max }$, of 5-CLSL, 5-PSSL, 5-PISL, 5-PASL, 5PGSL, 5-PESL, 5-PCSL, and 5-DGSL spin-labels can be used as empirical indicators of the selectivity of interaction. Binding of the protein induces significant increases in the values of $A_{\max }$ for all the spin-labels (Table II). Among the acidic lipids, $\mathrm{CL}^{-}$, $\mathrm{PS}^{-}$, and $\mathrm{PA}^{2-}$ interact more strongly with the basic protein than does $\mathrm{PG}^{-}$, whereas $\mathrm{PI}^{-}$and $\mathrm{PA}^{-}$interact less strongly. The zwitterionic lipids $\mathrm{PE}^{ \pm}$and $\mathrm{PC}^{ \pm}$show the smallest increases in $A_{\max }$ among the phospholipids. The neutral lipid DG exhibited the least protein-induced spectral effects. The order of selectivity based on these observations follows the sequence $\mathrm{PS}^{-}>\mathrm{CL}^{-}>\mathrm{PA}^{2-}>\mathrm{PG}^{-}>\mathrm{PI}^{-}>\mathrm{PA}^{-}>\mathrm{PE}^{ \pm}>\mathrm{PC}^{ \pm}>\mathrm{DG}$. This sequence of selectivity does not display an obvious relation to the size and conformation of the lipid headgroups, suggesting that it does not arise solely from either the interaction of the protein with groups other than the phosphate or a different location of the headgroup within the bilayer on binding of the protein.

The selectivity pattern may be analyzed more quantitatively if it is assumed, as was done above, that the ESR spectra of the C-5 labels correspond to fast exchange on the ESR time scale. The outer hyperfine splitting constant, $A_{\max }$, is then given by eq 1 . The fraction of spin-labeled lipids occupying the specific sites, $f$, is given by the equation for lipid-protein association appropriate for low spin-label concentrations (Brotherus et al., 1981; Knowles et al., 1979)

$$
f=\left[\left(n_{\mathrm{t}} / n_{\mathrm{p}}-1\right) / K_{\mathrm{r}}+1\right]^{-1}
$$

where $K_{\mathrm{r}}$ is the association constant of the labeled lipid relative to the unlabeled lipid (DMPG) and $n_{\mathrm{t}}$ is the total lipid/protein ratio. Combining eq 1 and 3 gives the relative association constant for the spin-labeled lipid.

$$
K_{\mathrm{r}}=\left(n_{\mathrm{t}} / n_{\mathrm{p}}-1\right)\left(A_{\max }-A^{\mathrm{f}}\right) /\left(A^{\mathrm{p}}-A_{\max }\right)
$$

Values of the relative association constants for the different spin-labeled lipids calculated in this way are given in Table III. For the purpose of this calculation, it was assumed that $A^{\mathrm{f}}$ is given by the value for the pure lipid, $n_{\mathrm{p}}=17$ and $A^{\mathrm{p}}=$ $A^{\mathrm{f}}+7.18 \mathrm{G}$, consistent with the parameters obtained from the least-squares fit to the data of 5-PCSL in Figure 7a. It is of interest that the value for $n_{\mathrm{p}}$ is quite close to the net positive charge, $Z=+20$, on the MBP. Only the value of $K_{\mathrm{r}}$ obtained for 5-PSSL is very sensitive to this choice of parameters, since its value for $A_{\max }$ lies very close to that for $A^{\mathrm{p}}$, and therefore small variations in the latter will produce a large effect on $K_{\mathrm{r}}$. Thus, the only conclusion that can be drawn for 5-PSSL is that the value of $K_{\mathrm{r}}$ must be much larger than that for the other lipids tested. 
The high selectivity for phosphatidylserine is particularly significant from the point of view of the lipid composition of the myelin membrane. Together with cerebroside sulfate, phosphatidylserine is the major negatively charged lipid and thus constitutes the principal binding site for the MBP. ${ }^{4}$ It is also significant that, of several phospholipids tested, the MBP displays the greatest binding to phosphatidylserine (Boggs \& Moscarello, 1978b) and, in particular, a greater extent of binding than found here for DMPG. For the other lipids studied here, the association constants relative to DMPG have rather modest values. None of them vary much from unity, indicating that the energetics for association of a single lipid with the MBP sites in bulk DMPG bilayers are not very different from those for phosphatidylglycerol itself. The net energy of association per protein is, of course, contributed mainly by the DMPG host lipids and the net binding energy is enhanced by an amount $Z e \Phi$, where $\Phi$ is the electrostatic surface potential of the bilayer.

It will be noted that the selectivities determined above refer to the different lipids in a DMPG host membrane, to which MBP binds strongly. However, certain of the selectivities of association correlate rather well with the binding of the protein to the different lipid species. The lipid/MBP binding stoichiometries have been found to be approximately $11,35,40$, and $70 \mathrm{~mol} / \mathrm{mol}$ for PS, PG, PA, and PE, respectively (Boggs \& Moscarello, 1978b). This corresponds to the order of selectivity of interaction for the spin-labeled lipid analogues given in Tables II and III. This must not necessarily be the case; for instance, there is relatively little binding of MBP to DMPC alone (although some effect is seen on $A_{\max }$; Figure 2).

$p H$ Dependence. The interfacial $\mathrm{p} K_{\mathrm{a}}$ 's of acidic lipid headgroups are shifted from the values expected in bulk solution by an amount $\Delta \mathrm{p} K^{\mathrm{int}}$, which depends both on the interfacial polarity and on surface electrostatics [see for reviews, Träuble (1976) and Cevc and Marsh (1987)]. In the presence of protein, there will be an additional contribution arising from the differential interaction with the two titrating forms, $\mathrm{L}$ and $\mathrm{LH}$, of the lipid. Thus, the net shift in $\mathrm{p} K_{\mathrm{a}}$ at the interface is given by

$$
\Delta \mathrm{p} K^{\mathrm{int}}=\Delta \mathrm{p} K^{\mathrm{pol}}+\Delta \mathrm{p} K^{\mathrm{el}}+\Delta \mathrm{p} K^{\mathrm{LP}}
$$

where the polarity-induced shift is $\Delta \mathrm{p} K^{\mathrm{pol}} \approx 1.1$ (Fernández \& Fromherz, 1977); the electrostatic shift is related to the surface potential, $\Phi$, by $\Delta \mathrm{p} K^{\mathrm{el}}=e \Phi / 2.3 k T$ [see, e.g., Cevc and Marsh (1987)]; and the shift due to lipid-protein interaction is $\Delta \mathrm{p} K^{\mathrm{LP}}=\log \left[K_{\mathrm{r}}(\mathrm{LH}) / K_{\mathrm{r}}(\mathrm{L})\right]$ (Marsh, 1988). 5-PASL has been found to titrate with a $\mathrm{p} K_{\mathrm{a}}$ of 7.4 in pure DMPC bilayers, corresponding to a polarity-induced shift of $\Delta \mathrm{p} K^{\mathrm{pol}}=0.9$ relative to the intrinsic $\mathrm{p} K_{\mathrm{a}}$ for the phosphate group (Horvăth et al., 1988). The present measurements yield a $\mathrm{p} K_{\mathrm{a} 2}$ of 9.3 for 5-PASL in DMPG bilayers, corresponding to an electrostatic shift of $\Delta \mathrm{p} K^{\mathrm{el}}=1.9$-comparable to that found in phosphatidic acid bilayers themselves which have been shown to titrate at a pH of 9.0 (Eibl \& Blume, 1979).

In the presence of MBP, the $\mathrm{p} K_{\mathrm{a}}$ shift of 5-PASL due to the lipid-protein interaction is estimated from the values of $K_{\mathrm{r}}(\mathrm{PA})$ given in Table III to be $\Delta \mathrm{p} K^{\mathrm{LP}}=\log \left[K_{\mathrm{r}}\left(\mathrm{PAH}^{-}\right)\right]$ $\left.K_{\mathrm{r}}\left(\mathrm{PA}^{2-}\right)\right]=-0.2$. The electrostatic shift is expected to be reduced by the neutralization of the surface charge on binding

\footnotetext{
${ }^{4}$ It is also of considerable interest to establish the selectivity pattern for spin-labeled analogues of lipids, such as sulfatide, cerebroside, of sphingomyelin, that are either unique or characteristic for the myelin membrane. This will be the subject of a further publication; a selectivity is observed for sulfatide that depends on whether the MBP is bound to unlabeled sulfatide or to DMPG (M. B. Sankaram, P. J. Brophy, G. Schwarzmann, K. Sandhoff, and D. Marsh, unpublished results).
}

of the protein. The maximum amount of this reduction is 1.9 $\mathrm{pH}$ units (i.e., the total electrostatic shift in the absence of protein). Estimates from electrostatic double-layer theory, using the net charge on the protein and the lipid-protein stoichiometry, suggest that this reduction may be closer to 0.5 $\mathrm{pH}$ units. The fact that the $\mathrm{p} K_{\mathrm{a}}$ in the presence of protein is very similar to that in the absence of protein therefore implies that the polarity shift induced on binding of MBP compensates the two downward shifts. The polarity shift is therefore increased by approximately $0.7 \mathrm{pH}$ unit on protein binding (the maximum being $2.1 \mathrm{pH}$ units). ${ }^{5} \quad$ This indicates that the polarity at the lipid-protein interface is significantly reduced relative to the uncomplexed lipid-water interface. Comparison with model experiments in solvents of different dielectric constant (Fernândez \& Fromherz, 1977) suggests that the lipid becomes partially dehydrated on binding of the protein.

\section{ACKNOWLEDGMENTS}

M.B.S. thanks the Alexander von Humboldt Foundation for a fellowship. The skillful technical assistance of $S$. Chatterjee in preparing the protein and of B. Angerstein in preparing various spin-labels is gratefully acknowledged.

Registry No. DMPG, 61361-72-6; DMPC, 18194-24-6.

\section{REFERENCES}

Boggs, J. M., \& Moscarello, M. A. (1978a) Biochim. Biophys. Acta 515, 1-21.

Boggs, J. M., \& Moscarello, M. A. (1978b) J. Membr. Biol $39,75-96$

Boggs, J. M., Moscarello, M. A., \& Papahadjopoulos, D. (1977) Biochemistry 16, 5420-5426.

Boggs, J. M., Moscarello, M. A., \& Papahadjopoulos, D. (1982) in Lipid-Protein Interactions (Jost, P. C., \& Griffith, O. H., Eds.) Vol. 2, pp 1-51, Wiley-Interscience, New York.

Boggs, J. M., Rangaraj, G., Moscarello, M. A., \& Koshy, K. M. (1985) Biochim. Biophys. Acta 816, 208-220.

Boggs, J. M., Chia, L. S., Rangaraj, G., \& Moscarello, M. A. (1986) Chem. Phys. Lipids 39, 165-184.

Braun, P. E. (1977) in Myelin (Morell, P., Ed.) pp 91-115, Plenum Press, New York.

Brotherus, J. R., Griffith, O. H., Brotherus, M. O., Jost, P. C., Silvius, J. R., \& Hokin, L. E. (1981) Biochemistry 20, 5261-5267.

Carrington, A., \& McLachlan, A. D. (1969) Introduction to Magnetic Resonance. With Applications to Chemistry and Chemical Physics, Harper and Row, New York.

Cevc, G., \& Marsh, D. (1987) Phospholipid Bilayers. Physical Principles and Models, Wiley-Interscience, New York.

Chao, I. P., \& Einstein, E. R. (1970) J. Neurochem. 17, 1121-1132.

Comfurius, P., \& Zwaal, R. F. A. (1977) Biochim. Biophys. Acta 488, 36-42.

Deibler, G. E., Martenson, R. E., \& Kies, M. W. (1972) Prog. Biophys. Biochem. 2, 139-165.

Eibl, H., \& Lands, W. E. M. (1969) Anal. Biochem. 30, $51-57$.

Eibl, H., \& Blume, A. (1979) Biochim. Biophys. Acta 553, $476-488$

\footnotetext{
${ }^{5}$ Similar conclusions can be reached from the titration of the 5-SASL stearic acid spin-label in the same system. In this latter case, the $\mathrm{p} K_{\mathrm{a}}$ of 5-SASL is increased from 8.0 to 9.6 on binding MBP, a result that can best be interpreted by a decrease in surface polarity accompanying dehydration induced by the protein (M. B. Sankaram, P. J. Brophy, W. Jordi, and D. Marsh, unpublished results).
} 
Epand, R. M., Moscarello, M. A., Zierenberg, B., \& Vail, W. J. (1974) Biochemistry 13, 1264-1267.

Eylar, E. H., \& Thompson, M. (1969) Arch. Biochem. Biophys. 129, 469-479.

Fernảndez, M. S., \& Fromherz, P. (1977) J. Phys. Chem. 81, 1755-1761.

Görrissen, H., Marsh, D., Rietveld, A., \& de Kruijff, B. (1986) Biochemistry 25, 2904-2910.

Horvâth, L. I., Brophy, P. J., \& Marsh, D. (1988) Biochemistry 27, 5296-5304.

Keana, J. F. W., Shimizu, M., \& Jernstedt, K. K. (1986) J. Org. Chem. 51, 2297-2299.

Knowles, P. F., Watts, A., \& Marsh, D. (1979) Biochemistry 18, 4480-4487.

Knowles, P. F., Watts, A., \& Marsh, D. (1981) Biochemistry 20, 5888-5894.

Lange, A., Marsh, D., Wassmer, K.-H., Meier, P., \& Kothe, G. (1985) Biochemistry 24, 4383-4392.

Liebes, L. F., Zand, R., \& Phillips, W. D. (1975) Biochim. Biophys. Acta 405, 27-39.

Lowry, O. H., Rosebrough, N. J., Farr, A. C., \& Randall, R. J. (1951) J. Biol. Chem. 193, 265-275.

MacNaughtan, W., Snook, K. A., Caspi, E., \& Franks, N. P. (1985) Biochim. Biophys. Acta 818, 132-148.

Marsh, D. (1982) Tech. Life Sci.: Biochem. B4/II, B426/ 1-B426/44.

Marsh, D. (1985) in Progress in Protein-Lipid Interactions (Watts, A., \& De Pont, J. J. H. H. M., Eds.) Vol. 1, pp 143-172, Elsevier, Amsterdam.

Marsh, D. (1988) in Supramolecular Structure and Function. Dubrovnik, Yugoslavia, Sept 16-28, 1987 (Pifat-Mrzljak, G., Ed.) pp 82-108, World Scientific Publishing, Singapore.

Marsh, D., \& Watts, A. (1982) in Lipid-Protein Interactions (Jost, P. C., \& Griffith, O. H., Eds.) Vol. 2, pp 53-126,
Wiley-Interscience, New York.

Mendz, G. L., Moore, W. J., Brown, L. R., \& Martenson, R. E. (1984) Biochemistry 23, 6041-6046.

Palmer, F. B., \& Dawson, R. M. C. (1969) Biochem. J. 111, 629-636.

Papahadjopoulos, D., Moscarello, M., Eylar, E. H., \& Isac, T. (1975) Biochim. Biophys. Acta 401, 317-335.

Ramsay, G., Prabhu, R., \& Freire, E. (1986) Biochemistry 25, 2265-2270.

Rietveld, A., Berkhout, T. A., Roenhorst, A., Marsh, D., \& de Kruijff, B. (1986) Biochim. Biophys. Acta 858, 38-46.

Rumsby, M. G. (1978) Biochem. Soc. Trans. 6, 448-462.

Rumsby, M. G., \& Crang, A. J. (1977) Cell. Surf. Rev. 4, 247-362.

Sachse, J.-H., King, M. D., \& Marsh, D. (1987) J. Magn. Reson. 71, 385-404.

Sankaram, M. B., Brophy, P. J., \& Marsh, D. (1989) Biochemistry 28, 9685-9691.

Sixl, F., \& Watts, A. (1982) Biochemistry 21, 6446-6452.

Somerharju, R., \& Wirtz, K. W. A. (1982) Chem. Phys. Lipids 30, 81-91.

Stone, A. L., Park, J. Y., \& Martenson, R. E. (1985) Biochemistry 24, 6666-6673.

Surewicz, W. K., Moscarello, M. A., \& Mantsch, H. H. (1987) Biochemistry 26, 3881-3886.

Träuble, H. (1976) in Structure of Biological Membranes (Abrahamsson, S., \& Pascher, I., Eds.) pp 509-550, Plenum Press, New York.

Watts, A., Harlos, K., Maschke, W., \& Marsh, D. (1978) Biochim. Biophys. Acta 510, 63-74.

Watts, A., Volotovski, I. D., \& Marsh, D. (1979) Biochemistry $18,5006-5013$.

Zwaal, R. F. A., Roelofsen, B., Comfurius, P., \& van Deenen, L. L. M. (1971) Biochim. Biophys. Acta 233, 474-479. 